\title{
Lysosomes and Their Role in Mucopolysaccharide Disorders: New Insights
}

\author{
Susanne G. Kircher ${ }^{1}$ Thomas Taylor ${ }^{2}$ \\ ${ }^{1}$ Center of Pathobiochemistry and Genetics, Medical University of \\ Vienna, Vienna, Austria \\ 2 Department of Orthopedics, SMZO - Donauspital, Vienna, Austria \\ Address for correspondence Susanne G. Kircher, MD, PhD, MBA, \\ Center of Pathobiochemistry and Genetics, Medical University of \\ Vienna, Waehringerstrasse 10, A-1090 Vienna, Austria \\ (e-mail: Susanne.kircher@meduniwien.ac.at).
}

J Child Sci 2018;8:e151-e155.

\begin{abstract}
Keywords

- mucopolysaccharide storage disease

- lysosome

- endosomes

- inflammation

- autophagy

Mucopolysaccharidoses (MPS) belong to the group of lysosomal storage diseases and are characterized by the deficiency of lysosomal enzymes involved in the degradation of glycosaminoglycans (GAGs). They are caused by inherited enzyme deficiencies that result from mutated genes producing defective enzymes. In MPS, the step-wise degradation process of GAG chains is impaired, and thus pieces of undegraded chains remain in the lysosomes impeding other processes in these cell organelles. Several enzymes-currently 12 are known-are necessary to degrade GAGs to sugars and small chemical substances, such as sulfate- or amino groups, to prepare them for excretion via exocytosis or to reintegrate them into new molecules by recycling. GAGs are major components of the connective tissue but play a role in shaping the vicinity of each cell surface as well; hence, any disturbance in their metabolism causes problems not limited to connective tissue but other tissues and organs. Therefore, MPS are multisystemic diseases involving many organs and restrict a wide range of organ functions. Organomegaly, typical skeletal changes known as "dysostosis multiplex," excessive storage in the nervous system with neurologic symptoms and impaired cognitive function have been known as the typical symptoms of the first classical MPS types for some time now. Because of new therapies and improved care, the life expectancy of MPS patients has increased significantly. Results of investigations that have only become available recently and new methods allowing for fine-meshed observation have revealed unexpected findings. These outcomes are not limited to excessive storage: patients show unpredictable reactions of the immune system, chronic inflammation, changes in metabolic pathways not directly related to the connective tissue, such as chronic arthritis, osteopenia, Parkinson-like symptoms, signs of dementia, and many more, which suggest that lysosomes have many more roles than just degradation of no longer needed macromolecules.
\end{abstract}

\section{Introduction}

Mucopolysaccharidoses (MPS) are a subgroup of the approximately 60 known lysosomal disorders. There are 12 enzyme deficiencies known to cause the different types of MPS, numbered from MPS I up to MPS IX. The first patients were described a hundred years ago with distinct faces and cognitive deficits, hearing impairment, and multiple skeletal changes, later defined as "dysostosis multiplex."1,2 In the middle of the last century, sugar-containing substances, the MPS, were received

May 31, 2018 accepted after revision June 30,2018
Issue Theme Advances in

Mucopolysaccharidoses; Guest Editors: Susanne G. Kircher, MD, PhD, MBA, Adriana M. Montaño, PhD, Christina Lampe, MD.

DOI https://doi.org/ 10.1055/s-0038-1669383. ISSN 2474-5871.
Copyright $\odot 2018$ Georg Thieme Verlag License terms KG Stuttgart · New York

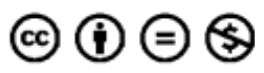


identified in the urine of patients, which were recognized as sugar chains with residues composed of amino groups and acid sulfate- or acetic acid groups. With simple tests, such as the Berry-spot test, their increased excretion in the urine of patients could be detected. ${ }^{3-5}$ Correspondingly excessive storage in the lysosomes could be observed under the microscope to further support the diagnosis of an MPS disease, for instance, with metachromatic vacuoles in granulocytes of peripheral blood smears. ${ }^{6}$ In the 1960 s, Neufeld and Cantz recognized cell lines which corrected the pathological storage in the lysosomes by transfer of unidentified substances in the culture media, the so-called cross-correction by correcting factors, which they numbered systematically. ${ }^{7}$ Soon after, they identified these factors as proteins, enzymes, degrading substances in the lysosomes. ${ }^{8-10}$ From that moment on it was clear that MPS were caused by deficient enzymes in the lysosomes, giving rise to excessive storage of nondegraded material in the lysosomes, which was thought to be a digesting cell organelle, a waste bag, the "cell stomach."

\section{Lysosomes and Their Discovery}

Sixty years ago, the lysosomes were discovered by de Duve. ${ }^{11}$ He and his colleagues worked on the liver enzyme glucose-6phosphate, its action on insulin, and its connection to the cell fraction he called the "microsomes," consisting of membranes found, for example, in the endoplasmic reticulum. In these experiments, a nonspecific acid phosphatase was observed to have a very different behavior. It was localized within sac-like particles surrounded by a membrane, which after centrifugation was found not only in the mitochondrial fraction but also in the microsomal fraction. He named the fraction "L" for light-mitochondrial particles gained by fivefraction centrifugation protocol. In 1955, there were five more enzymes identified in this fraction. Four of them were recognized as hydrolases, situated in these L-structures with digestive function, whereas a fifth enzyme was confirmed as constituent of peroxisomes (urate oxidase). ${ }^{11}$

de Duve proposed the name lysosomes for these cell organelles and recognized that they were filled with enzymes, mainly acid hydrolases, which could perform specific degradations in the acidic milieu of lysosomes, membrane-bound cell organelles, that are found in human and animal cells. ${ }^{11}$ In 1963, the first international symposium on lysosomes brought together many groups working on the field of lysosomes. At that time, the possible involvement of lysosomes in various pathological processes was suspected.

Lysosomal enzymes (many of them are hydrolases) can break down a wide variety of macromolecules and cell deposits, which are delivered to the lysosomes via endocytosis, phagocytosis, and autophagy. Their role is to digest substances into their smallest constituent molecules to recycle them into newly created macromolecules or to excrete them via exocytosis. ${ }^{11}$

Lysosomes constitute about $5 \%$ of the cell volume and can differ in shape and size. Any disturbance of the degradation processes, irrespective of which macromolecules are involved, leads to an increased size and number of these cell organelles, which can be representative of the disease. This is seen in all
MPS, as well as in related disorders such as glycoproteinoses (i.e., fucosidoses, $\alpha$-mannosidoses, aspartylglucosaminuria), neurolipidoses (i.e., Sandhoff disease, Niemann-Pick disease, Tay-Sachs disease) or in Gaucher disease, Fabry disease, and numerous other lysosomal storage disorders (LSDs). Many of them exhibit distinct features of the deposited material, such as foamy cells or sea-blue histiocytes in Niemann-Pick disease, Gaucher cells with striped cytoplasma, large foamy histiocytes in Wolman's disease, Gasser cells with purplish, metachromatic or Alder granules in MPS, periodic acid-Schiff (PAS) staining-positive vacuoles in fucosidosis, or curvilinear bodies in Batten's disease. 6

\section{Lysosomes and Mucopolysaccharidoses}

The group of MPS disorders is comprised of 12 different enzyme deficiencies, most of them affecting hydrolases involved in the degradation process of glycosaminoglycan (GAG) chains. All involved lysosomal enzymes are produced by the endoplasmic reticulum and are subsequently glycosylated in the Golgi apparatus, where they are phosphorylated with a mannose-6-phosphate (M6P) residue as recognition marker. ${ }^{12}$ This marker enables the enzymes to be transported from the trans-Golgi network into the lysosomes via a mannose-6-phosphate receptor (M6PR) situated in the lysosomal membrane. Once there, hydrolases remove the M6P residue, which may be recycled to the trans-Golgi network to bind newly synthesized enzymes. The lysosomal membrane in total contains more than a hundred proteins responsible for the trafficking between lysosomes and the cytoplasm of the cell. The membrane has many functions, such as chaperone-mediated autophagy, transport of the hydrolases into the lysosome, and contains transporters and channels for amino acids, sugars, and lipids.

\section{Enzyme Deficiency as Cause of Mucopolysaccharidoses}

Each of the GAG degradation steps is performed by a specific lysosomal enzyme. ${ }^{12}$ If one of the enzymes has reduced activity or is missing, the process of digestion necessarily ends prematurely and none of the following steps will be performed. The cause of lysosomal enzyme deficiencies relies on the presence of different types of mutations in their respective genes. One possible outcome is a missense mutation, which leads to the exchange of a single amino acid in the protein chain. This can lower enzyme activity, limit half-life of the enzyme and thus its availability, make it vulnerable to the acidic milieu in the lysosome, and cause it to be digested sooner. In MPS, enzyme activity is typically lowered but residual activity remains. Other types of mutations such as stop-mutations, deletions, or duplications are responsible for a usually significant shortening of the protein chain, which cannot be processed in the Golgi apparatus or transported to the lysosome and/or can be eliminated by autophagy. In this case, no residual enzyme activity can be measured.

With the exception of the X-linked MPS II (Hunter's disease), all other MPS types are inherited by an autosomal recessive 
trait. Patients with the recessive forms of MPS have a high number of different gene mutations which affect in many combinations both alleles and the amount of the produced enzyme proteins. Therefore, a wide range of enzyme activity may be found, from zero to several percent of the normal activity, which explains the wide spectrum of clinical severity one can observe from most severely affected patients with first signs in utero to attenuated forms of MPS in adulthood.

GAGs are not only the main ingredient of the extracellular matrix of the connective tissue but also necessary for the proteoglycan chains of the glycocalyx of cell surfaces. ${ }^{12}$ Depending on their localization, their composition varies but is quite often distinctive for a specific tissue or organ. GAGs are heparan sulfate (HS), dermatan sulfate (DS), chondroitin sulfate (CS), keratan sulfate (KS), and hyaluronic acid (HA). ${ }^{13}$ Sometimes, lysosomal enzymes can catalyze reactions in different types of GAG chains, for instance, the deletion of the sugar iduronic acid in HS as well as DS, as in MPS I, MPS II, or MPS VI. ${ }^{14}$ Other lysosomal enzymes only involve the degradation process of HS (MPS III) or KS (MPS IV). ${ }^{15,16}$ Any enzyme deficiency leads to an accumulation and storage of fragments of the involved GAG chain(s), an increase in lysosomes in size and number, as well as cell size. In the long run, this damages cells and causes apoptosis; thus, all the accumulated fragments will enter the bloodstream and be excreted into the urine of patients. These fragments can help to identify the chemical properties of the excessively stored material, allow to identify the type of MPS, and act as a biomarker. ${ }^{15-17}$

\section{Lysosomes and Other Functions}

In 1983, de Duve redefined the lysosomes as polymorphic particles with differing size, shape, and internal structure. ${ }^{18}$ New discoveries regarding the cell surface were the observation of receptors/ligands and the receptor-mediated fluidphase nonselective endocytosis (pinocytosis, protocytosis) or phagocytosis. In this process, material is first incorporated by temporary storage in inactive vacuoles (first named as receptosomes or endosomes), which subsequently fuse with lysosomes or regurgitate their contents out of the cell. ${ }^{18}$ The sorting process, which determines what substances should be incorporated into a cell and transported to/fused with the lysosome, is not only regulated by the cell surface as well as endosomes, but also influenced in part by (1) the lysosomes, as they are involved in membrane recycling, and (2) somewhat indirectly via the Golgi apparatus. Membranes are necessary for all the vesicles involved in the digestion processes and may be reused again. There is evidence that lysosomes are no longer the place of no return for incorporated membrane material. However, the amount of recycled membrane material is probably not very high. It was observed that heavily vacuolated cells may be immobilized by high amounts of membrane material, which can interfere with the recycling process.

Lysosomes help to degrade endocytosed extracellular material as well as intracellular substances sequestered by autophagy. In the latter case, lysosomes fuse and form auto- lysosomes and phagolysosomes. ${ }^{19}$ Many proteins are involved in this machinery of trafficking, interacting, and maintaining the charge difference of ions between the cellular cytoplasm and lysosomes. Lysosomes can fuse with the plasma membranes of the cells to repair them or defend against parasites too big to be tackled by single cells. The integrity of the lysosomal membrane is also protecting the cell from autolytic processes. But lysosomal overloading with stored material can induce lytic properties against cellular structures and result in apoptosis, necrosis, and cell death.

\section{Lysosomes and Cell Signaling}

The cytosolic site of the lysosomal membrane is involved in signaling complexes, especially the mammalian target of rapamycin complex 1 (mTORC1) and small GTP-ases of the RAG family. The mTORC1 complex regulates the cellular metabolism arising from nutrients, energy, and growth factors. As part of this complex, mTOR can influence the transcription factor EB (TFEB) via phosphorylation, which in turn influences the transcription of genes encoding lysosomal proteins leading to an upregulation. TFEB plays an important role in the exocytosis pathway of lysosomes, as they move closer to and fuse with the plasma membrane to excrete their contents, removing stored material from the cell amounting to a rescue from lysosomal overloading. ${ }^{20}$ For this process, TFEB induces an increase in intracellular calcium levels through the activation of the lysosomal calcium-channel Mucolipin 1. This physiological cell-saving and clearing function can reduce accumulation of pathogenic stored proteins as could be observed in experiments for Huntington's disease or Parkinson's disease. ${ }^{21}$ Protein aggregates in these two diseases as well as Alzheimer's disease can cause severe neurodegenerative disorders and it appears to be a promising therapeutic approach to clear the cell via lysosomal autophagy, which will then degrade and excrete the aggregates, rescuing the cell from any excessively stored material. ${ }^{22}$

\section{Autophagy}

Autophagy is an essential and physiological process for homeostasis in each cell and depends on lysosomes. Autophagy declines with age but also in disease, for instance, LSDs. $^{23}$ Excessive storage of material in the lysosomes impairs the process of autophagy in each cell, disturbs the elimination of damaged cell components, aggregated proteins, and other components; hampers any recycling processes; and diminishes energy metabolism and homeostasis in each cell. ${ }^{24}$ Selective autophagy, such as aggrephagy, is responsible for clearing cells of misfolded proteins, whereas mitophagy and ribophagy take care of dysfunctional mitochondria or defective ribosomes. Lipophagy removes lipid droplets, with reduced clearance observed in atherosclerosis or hepatic steatosis. Glycophagy degrades accumulated glycogen and xenophagy prevents cell damage by removing pathogens such as viruses, bacteria, and parasites. Mitophagy also reduces DNA damage as it suppresses the oxidative stress by reducing mitochondrial reactive oxygen species ${ }^{25}$ All these cellular autophagy steps are promoting cell 
survival and should be understood as a process for better cell survival.

Although in LSDs the underlying gene defects, the impaired metabolic pathways, the stored material, and the speed of accumulation may vary, the result looks the same: the cell clearance and autophagy are reduced, energy and homeostasis are impaired, and survival of cells is endangered. Several late-onset neurodegenerative disorders and neuronopathic LSDs, such as neuronal ceroid lipofuscinoses, sphingolipidoses, or glycogenosis type II (Pompe's disease) have shown to be very susceptible to effects on lysosomal storage and defective autophagy. ${ }^{23}$ This might be explained by the high metabolic requirements of neurons, their shape and polarization, the protein-clearing processes in these long-lived cells, or the disturbed function in myocytes due to vacuoles filled with stored material.

\section{Lysosomes and Their Role in Drug Uptake}

The accumulation of stored material in lysosomes can result not only from defective degrading enzymes but also from disturbed transport function of transporter proteins or channels in the lysosomal membrane. Independent of the underlying cause, the function of lysosomes, and sooner or later of other cell organelles as well, is impaired, leading to reduced autophagy, induced inflammation, and altered calcium homeostasis. Therefore, an enhanced clearance by TFEB overexpression could restore the defective cell metabolism. The enhancement of hydrolases synthesis and maturation might help to slow down or even reverse the storing process.

Often in LSDs, the intralysosomal ion concentration is altered and the lysosomal pH-value is aberrant. This has additional effects within the lysosomes, such as altered activity of all enzymes as they depend on the acidic milieu, as well as reduced intracellular autophagy and impaired cellular trafficking. To modulate the cellular and lysosomal calcium levels might be an efficient strategy to influence degradation and clearance of accumulated macromolecules. ${ }^{26}$

Substances bound to the cell surface are taken up by endocytosis and delivered to the lysosomes for digestion in most cases. Packing and conformation of these substances may play a role in their effective degradation by the lysosomal milieu. There is clear evidence that under physiological conditions, lysosomal enzymes are synthesized in the endoplasmic reticulum and marked with M6P. There are two different M6P receptors identifying the M6P residue, designated MPR46 and MPR300. Usually, M6P containing enzymes are transported via clathrin-coated vesicles to the endosomes, where the acidic milieu induces the dissociation of the M6P-ligand complex which is then recycled to the transGolgi network or to the cell surface. But approximately 5 to $20 \%$ of the newly synthesized enzymes escape this cell sorting mechanism and are secreted from the cell into the extracellular space from where they may be recaptured again by M6PR on the cell surface and transported to the lysosomes. ${ }^{27}$ This effect was the basis of the experiments of Neufeld and coworkers: The escape of enzymes from cells of one MPS type and their recapture by the M6P receptors on the cell surface of cells with a different MPS type, and therefore a different enzyme defect, resulted in the reciprocal cross-correction of excessive storage in the combined cell culture of fibroblasts derived from different MPS patients. ${ }^{7,8}$ The same basic principle is used in enzyme replacement therapy (ERT). Patients get periodical enzyme-rich infusions of recombinant, synthesized enzymes which can be captured by receptors on cell surfaces and incorporated into cells and lysosomes, where they alleviate enzymatic deficiencies and help diminish stored material.

Besides these well-known pathways for lysosomal trafficking of hydrolytic enzymes, there is clear evidence that there are alternate, M6P-independent pathways for lysosomal enzyme sorting. Markmann et al showed that non-phosphorylated enzymes (without M6P) were secreted from cells and could be recaptured via low-density lipoprotein (LDL) receptor and LDL receptor-related protein 1 , which might impact on improving efficiency of ERT in different LSDs. Furthermore, other sugar receptors on cell surfaces, such as asialoglycoprotein, mannose, or fucose receptors, might play a role in the uptake of lysosomal enzymes. ${ }^{27}$

\section{Lysosomal Storage Disorders as Inducers of Inflammation}

Immune system abnormalities and irregularities are observed in LSDs. There are various abnormalities, such as increased production of autoantibodies (Gaucher disease, Fabry disease), increased immune-mediated reactions in MPS, or an inappropriate activation of microglia in neurodegenerative foci of Niemann-Pick disease. ${ }^{28}$ The impaired cell metabolism in LSDs leads to disturbances in autophagy, impaired autophagosomelysosome fusion, accumulation of undegraded macromolecules in the cell, dysfunction of other cell organelles such as mitochondria, and manifests with increased expression of chemokines/cytokines, increased signs of inflammation and neovascularization. Especially in Gaucher disease, the consistently increased levels of proinflammatory cytokines and chemokines as well as the chronic stimulation of the immune system may be responsible for the production of monoclonal and polyclonal immunoglobulins, which result in the development of multiple myeloma in these patients. ${ }^{28}$

The accumulation of GAGs in MPS starts a cascade of intracellular responses, such as metabolic, inflammatory, and immunological effects in several organs. Especially the immune system and the skeleton share some of the same regulatory molecules, such as classical cytokines, receptor proteins, adaptor proteins, transcription factors, and signal transducers influencing the interplay between bone-forming osteoblasts and bone resorbing osteoclasts, which can be observed in severe changes of bone formation and rheumatoid-like chronic arthritis of many joints with increasing stiffness and contractures. The mechanism of the aberrant activation of neuroinflammatory responses in those MPS types with predominant HS storage is not yet fully understood, but in animal models with MPS type IIIB the administration of oral corticosteroids as immunosuppressive therapy was noticed to be beneficial. ${ }^{28}$ 


\section{Conclusions}

This review only partially highlights some of the currently held views of LSDs and their multisystemic consequences, but should give a glimpse of the complex function of lysosomes in the cell. It is no longer considered just a "bag for the digestion" of undegraded and therefore stored material, but has been shown to be a crucial component in many processes such as autophagy, immune response, cell energy metabolism, oxidative stress, and many more. There appears to be great potential to develop therapies and administer drugs on multilevel targets to improve the clinical signs and symptoms of patients with MPS. Successful stem cell transplantation or the continuous administration of ERT might improve some of the clinical symptoms, but the future in therapy will be as multisystemic and complex as the MPS themselves are.

\section{Conflict of Interest}

None.

\section{References}

1 Hunter C. A rare disease in two brothers. Proc R Soc Med 1917;10 (Sect Study Dis Child):104-116

2 Hurler G. Über einen Typ multipler Abartungen, vorwiegend am Skelettsystem. Z Kinderheilkd 1920;24:220-234

3 Berry HK. Screening for mucopolysaccharide disorders with the Berry spot test. Clin Biochem 1987;20(05):365-371

4 Calatroni A. Test for mucopolysaccharidoses: simple method for quantitative estimation of urinary glycosaminoglycans. Clin Chem 1972;18(03):266-269

5 Whiteman P. The quantitative determination of glycosaminoglycans in urine with Alcian Blue 8GX. Biochem J 1973;131(02): 351-357

6 Hann IM, Lake BD, Lilleyman J, Pritchard J. Storage disorders. In: Colour Atlas of Paediatric Haematology. 3rd ed. Oxford, New York, Toronto: Oxford University Press; 1996:148-171

7 Neufeld EF, Cantz MJ. Corrective factors for inborn errors of mucopolysaccharide metabolism. Ann N Y Acad Sci 1971;179: 580-587

8 Fratantoni JC, Hall CW, Neufeld EF. Hurler and Hunter syndromes: mutual correction of the defect in cultured fibroblasts. Science 1968;162(3853):570-572

9 Fratantoni JC, Hall CW, Neufeld EF. The defect in Hurler and Hunter syndromes. II. Deficiency of specific factors involved in mucopolysaccharide degradation. Proc Natl Acad Sci U S A 1969; 64(01):360-366
10 Dorfman A, Matalon R. The Hurler and Hunter syndromes. Am J Med 1969;47(05):691-707

11 de Duve C. The lysosome turns fifty. Nat Cell Biol 2005;7(09): 847-849

12 Neufeld E, Muenzer J. The Mucopolysaccharidoses. In: Scriver CBA, Sly S, et al., eds. The Metabolic and Molecular Bases of Inherited Disease. 8th ed. New York: McGraw-Hill; 2001:3421-3452

13 Meyer K. Biochemistry and biology of mucopolysaccharides. Am J Med 1969;47(05):664-672

14 McKusick VA. The nosology of the mucopolysaccharidoses. Am J Med 1969;47(05):730-747

15 Tomatsu S, Gutierrez MA, Ishimaru T, et al. Heparan sulfate levels in mucopolysaccharidoses and mucolipidoses. J Inherit Metab Dis 2005;28(05):743-757

16 Tomatsu S, Okamura K, Maeda H, et al. Keratan sulphate levels in mucopolysaccharidoses and mucolipidoses. J Inherit Metab Dis 2005;28(02):187-202

17 Kircher SG, Bajbouj M, Miebach E, Beck M. Mucopolysaccharidoses - A Guide for Physicians and Parents. London, Boston: UNIMed Science Verlag Bremen International Medical Publishers; 2007

18 de Duve C. Lysosomes revisited. Eur J Biochem 1983;137(03): 391-397

19 Wartosch L, Bright NA, Luzio JP. Quick guide lysosomes. Curr Biol 2015;25:R301-R327

20 Medina DL, Fraldi A, Bouche V, et al. Transcriptional activation of lysosomal exocytosis promotes cellular clearance. Dev Cell 2011; 21(03):421-430

21 Sardiello M, Palmieri M, di Ronza A, et al. A gene network regulating lysosomal biogenesis and function. Science 2009;325 (5939):473-477

22 Saftig P, Haas A. Turn up the lysosome. Nat Cell Biol 2016;18(10): 1025-1027

23 Seranova E, Connolly KJ, Zatyka M, et al. Dysregulation of autophagy as a common mechanism in lysosomal storage diseases. Essays Biochem 2017;61(06):733-749

24 Ballabio A, Gieselmann V. Lysosomal disorders: from storage to cellular damage. Biochim Biophys Acta 2009;1793(04):684-696

25 Ryter SW, Bhatia D, Choi ME. Autophagy: alysosome-dependent process with implications in cellular redox homeostasis and human disease. Antioxid Redox Signal 2018. doi: 10.1089/ ars.2018.7518

26 Appelqvist H, Wäster P, Kågedal K, Öllinger K. The lysosome: from waste bag to potential therapeutic target. J Mol Cell Biol 2013;5 (04):214-226

27 Markmann S, Thelen M, Cornils K, et al. Lrp1/LDL receptor play critical roles in mannose 6-phosphate-independent lysosomal enzyme targeting. Traffic 2015;16(07):743-759

28 Rigante D, Cipolla C, Basile U, Gulli F, Savastano MC. Overview of immune abnormalities in lysosomal storage disorders. Immunol Lett 2017;188:79-85 